\title{
EP-189
}

\section{Pancreatic angiosarcoma: A case report}

\author{
Farah Najla SUPANDI ${ }^{* 1,2}$, Balraj SINGH', Haritharan THAMUTARAM ${ }^{3}$, Kin Foong LIM ${ }^{4}$, Ahmad Ramzi YUSOFF ${ }^{1,2}$ \\ 'Department of HPB and Liver Transplantation, Hospital Selayang, Selangor, Malaysia \\ ${ }^{2}$ Department of Surgery, Faculty of Medicine, Universiti Teknologi MARA, Sungai Buloh, Malaysia \\ ${ }^{3}$ General and Hepatobiliary Surgery, Subang Jaya Medical Centre, Subang Jaya, Malaysia \\ ${ }^{4}$ General and Hepatobiliary Surgery, Pantai Hospital, Kuala Lumpur, Malaysia
}

Introduction: Pancreatic cancer has a relative 5-year survival of less than $10 \%$. The most common neoplasm of the pancreas is ductal adenocarcinoma, which comprises $85 \%$ of all malignant pancreatic tumours. Primary pancreatic sarcomas are extremely rare which account for less than $0.1 \%$ of all pancreatic malignancies and pancreatic angiosarcomas attribute to $1 \%$ of all tissue sarcomas. Sarcomas of the pancreas are more aggressive and have a dismal prognosis.

Methods: A 69-year-old lady presented with obstructive jaundice, abdominal discomfort and associated constitutional symptoms. She was investigated and found to have obstructive jaundice with normal tumor marker assays. An endoscopic ultrasound was done followed by a needle biopsy which confirmed a pancreatic head angiosarcoma followed by an ERCP and a stent placement. A CT abdomen done revealed a pancreatic head and uncinate tumor with a stent in the common bile duct.

Results: Patient underwent a pylorus preserving pancreaticoduodenectomy with an uneventful post-operative recovery. She defaulted her oncology appointments and was followed up with serial imaging. She developed a local recurrence 18 months after surgery and succumbed to her disease after 3 years.

Conclusions: Pancreatic angiosarcoma is an aggressive tumor compared to other pancreatic malignancies. For a definite diagnosis of angiosarcoma, histopathologic and immunohistochemical analysis are necessary. Surgical resection offers the only possible cure, while oncological treatment has variable outcome. Currently, there are no treatment protocols available due to the small number of cases present in literature. 